\title{
FINITE DESCENT OBSTRUCTION FOR CURVES OVER FUNCTION FIELDS
}

\author{
JOSÉ FELIPE VOLOCH
}

\begin{abstract}
We prove that a form of finite Galois descent obstruction is the only obstruction to the existence of integral points on integral models of twists of modular curves over function fields.
\end{abstract}

\section{INTRODUCTION}

The purpose of this paper is to study finite descent obstructions for integral points on curves over function fields. In the special case of modular curves, we use a similar approach to the work of D. Helm and the author [5], where we obtained results over $\mathbf{Q}$ from Serre's conjecture. In this paper, we replace the use of Serre's conjecture with Deligne's [2] approach to modularity of elliptic curves over function fields. We prove that the existence of an adelic point surviving finite descent obstructions given by covers coming from modular curves of higher levels (and satisfying an additional non-integrality condition at some place, in certain situations to be made precise below) implies the existence of a global point. We also show that this additional condition cannot be removed and, without it there are counterexamples.

Let $K$ be a global field and $X, Y$ be smooth $K$-varieties. Let $\pi: Y \rightarrow$ $X$ be an etale map of varieties such that $\pi: \bar{Y} \rightarrow \bar{X}$ is Galois (where here and elsewhere, the bar denotes base change to the algebraic closure $\bar{K}$ of $K)$. As usual, a twist of $\pi$ is a map $\pi^{\prime}: Y^{\prime} \rightarrow X$, defined over $K$, such that $\pi^{\prime}: \bar{Y}^{\prime} \rightarrow \bar{X}$ is isomorphic over $\bar{K}$ to $\pi: \bar{Y} \rightarrow \bar{X}$ as a cover. The set of isomorphism classes of twists of $\pi$ will be denoted $T w(\pi)$. Let $S$ be a finite set of places of $K$ and $\mathcal{O}_{S}$ the ring of $S$-integers of $K$. Let $\mathcal{X}, \mathcal{Y}$ and so on denote integral models of $X, Y$ over $\operatorname{Spec} \mathcal{O}_{S}$. Standard descent theory ([8]) gives the following:

$$
\mathcal{X}\left(\mathcal{O}_{S}\right)=\cup_{\pi^{\prime} \in T w(\pi)} \pi^{\prime}\left(\mathcal{Y}^{\prime}\left(\mathcal{O}_{S}\right)\right) .
$$

2000 Mathematics Subject Classification. Primary 11G30; Secondary 14G25.

Key words and phrases. descent obstruction, modularity, galois representation, function fields. 
Indeed, for $P \in \mathcal{X}\left(\mathcal{O}_{S}\right), \pi^{-1}(P)$ is a torsor of the Galois group of $\pi$ which determines a $\pi^{\prime}$. We can even restrict the union to a subset of $T w(\pi)$, described by local conditions, which is finite if the degree of $\pi$ is prime to the characteristic of $K$. This is a consequence of the Chevalley-Weil theorem.

Let $\left(P_{v}\right) \in \prod_{v \notin S} \mathcal{X}\left(\mathcal{O}_{v}\right) \times \prod_{v \in S} X\left(K_{v}\right)$. If there exists $\pi^{\prime} \in T w(\pi)$ such that the twisted torsor $\mathcal{Y}^{\prime}$ contains a point $\left(Q_{v}\right) \in \prod_{v \notin S} \mathcal{Y}^{\prime}\left(\mathcal{O}_{v}\right) \times$ $\prod_{v \in S} Y^{\prime}\left(K_{v}\right)$ that maps to $\left(P_{v}\right)$, we say that $\left(P_{v}\right)$ is unobstructed by the cover. This differs slightly from the convention in [5] by the inclusion of the places in $S$. There, the conditions at $S$ were irrelevant, but will be relevant here. Global points are thus unobstructed. A natural question is to identify those adelic points that are unobstructed by all Galois covers which we denote by $\mathcal{X}^{f-c o v}$ following [9], who conjectured that if $X$ is a projective curve over a number field, then $\mathcal{X}^{f-\operatorname{cov}}=X(K)$ (note that integrality conditions don't matter in the projective case). A stronger conjecture in the number field case is that abelian covers is enough. This stronger conjecture, in the number field case, is equivalent to whether the Brauer-Manin obstruction is the only obstruction to the existence of rational points, see $[8,7]$. Poonen and the author [6] proved that the Brauer-Manin obstruction is the only obstruction to the existence of rational points in the function field case under fairly general additional hypotheses and some other cases were proved in [10]. However, in the function field case, it is not true that the Brauer-Manin obstruction is equivalent to the finite abelian descent obstruction, since the former allows for some flat inseparable covers and, indeed, the proofs in $[6,10]$ make extensive use of covers coming from inseparable isogenies in the curve's Jacobian. Thus, the results of this paper and of $[6,10]$ are related but neither supercedes the other. We note also that, in work in preparation, the author and D. Harari prove that the finite abelian descent obstruction is enough for affine varieties over function fields. However, this last paper uses Artin-Schreier covers and those typically do not satisfy finiteness of the relevant subset of $T w(\pi)$ alluded to above. In the present paper, our covers are typically of degree prime to the characteristic, so this finiteness holds for them.

\section{Modular CuRves}

Let $N$ be a positive integer and $Y_{N} / \mathrm{Q}$ be the affine curve parametrizing triples $(E, P, C)$ where $E$ is an elliptic curve, $P$ is a point on $E$ of exact order $N$ and $C$ is a cyclic subgroup of $E$ of order $N$ such that $P$ and $C$ generate $E[N]$. $Y_{N}$ has a smooth model over $\mathbf{Z}[1 / N]$. Let $X$ be a twist of some $Y_{m}$. As in [5] we obtain etale Galois covers of $X$ 
by considering modular curves with additional level structure. In [5] it was assumed that $m>3$ whereas in Theorem 1 below we only assume $m>1$. What is required is that the natural maps $Y_{N} \rightarrow Y_{m}$ for $m \mid N$ be ramified only at the cusps and, for that, $m>1$ suffices. Again, as in [5] we conclude that a point of $\mathcal{X}^{f-c o v}$ consists of elliptic curves $E_{v} / K_{v}$ with good reduction for $v \notin S$ for which, for all primes $\ell$, there exist Galois representations $\rho_{\ell}: G_{K} \rightarrow G L_{2}\left(\mathbf{Z}_{\ell}\right)$, such that $\rho_{\ell}$ restricted to a decomposition group at $v$ corresponds to the action of $G_{K_{v}}$ on the Tate module $T_{\ell} E_{v}$. In particular, these representations are all unramified outside $S$ and their determinant is the cyclotomic character.

Here is our first result.

Theorem 1. Assume that $K$ is a global function field. Let $\mathcal{X}$ be the $S$-integral model of a twist of $Y_{m}, m>1$ corresponding to a representation $\bar{\rho}: G_{K} \rightarrow G L_{2}(\mathbf{Z} / m)$. We have that, if $\mathcal{X}^{f-c o v}$ has a point with multiplicative reduction at some place in $S$ (when viewed as an elliptic curve) then $\mathcal{X}\left(\mathcal{O}_{S}\right)$ does too.

Remark 2.1. The hypothesis on the existence of a place with multiplicative reduction in the theorem is automatically satisfied if $\bar{\rho}$ is ramified and semistable at some place of $K$.

Proof. The existence of a point in $\mathcal{X}^{f-c o v}$ yields (see [5]) a Galois representation $\rho: G_{K} \rightarrow G L_{2}\left(\mathbf{Z}_{\ell}\right)$ for some prime $\ell \neq p$ extending the representation obtained from $\bar{\rho}$ to $G L_{2}\left(\mathbf{Z} / \ell^{d}\right)$, where $\ell^{d} \| m$. We will follow [2] to obtain an elliptic curve from $\rho$. The representation $\rho$ and its twists by characters of $G_{K}$ give rise to $L$-functions which are rational functions in $q^{-s}$ and satisfy functional equations ([2], section 9.1). This implies that the $L$-function associated to $\rho$ comes from a Drinfel'd modular form, which is cuspidal if this $L$-function is a polynomial in $q^{-s}$. Deligne gives a necessary and sufficient condition for that to happen which we will recall below. When this condition is fulfilled, the modular form is a cusp form and gives rise to an elliptic curve occurring as a factor of the Jacobian of a Drinfel'd modular curve and whose $\ell$-adic representation is $\rho$. This elliptic curve provides us with a point in $\mathcal{X}\left(\mathcal{O}_{S}\right)$, because its Galois representation is $\rho$ which is unramified outside of $S$. Moreover, since its $L$-function is a polynomial, it has multiplicative reduction at some place, which then must be in $S$.

Let $G_{0}$ be the subgroup of $G_{K}$ which acts trivially on constants. Denote by $V$ the 2-dimensional $\mathbf{Q}_{\ell^{-}}$-vector space with $G_{K}$-action given by $\rho$. Denote by $\rho_{0}$ the restriction of $\rho$ to $G_{0}$, but we abuse notation and still denote by $V$ the space where $G_{0}$ is acting. Deligne's conditions for the $L$-function to be polynomial are that the invariants $V^{G_{0}}$ and coinvariants $V_{G_{0}}$ both vanish. We proceed to verify these conditions 
under the assumptions of the theorem. The verification is the same for $\rho$ or its twists, so we do it just for $\rho$.

First, since $\rho$ is everywhere locally the action of Galois on the Tate module of an elliptic curve, its determinant is the cyclotomic character. Therefore, the determinant of $\rho_{0}$ is 1 . At a place $v$ of multiplicative reduction (which is automatically split multiplicative when we extend scalars to the algebraic closure of the constant field), the curve $E_{v}$ is a Tate curve $\mathbf{G}_{m} / q^{\mathbf{Z}}$ and the local Galois action on $\ell^{n}$-torsion has a generator $\sigma: \zeta_{\ell^{n}} \mapsto \zeta_{\ell^{n}}, q^{1 / \ell^{n}} \mapsto \zeta_{\ell^{n}} q^{1 / \ell^{n}}$. In particular, $V_{G_{0}} \neq V$. If $V_{G_{0}}$ is one-dimensional, then there is a one dimensional subspace $W$ of $V$, which is a $G_{0}$ submodule and $V_{G_{0}}=V / W$. Now, $V_{G_{0}}$ is a trivial $G_{0}$-module, so the action of $G_{0}$ on $W$ is through $\operatorname{det} \rho_{0}=1$, i.e., $W$ is a trivial $G_{0}$-module, hence $W=V^{G_{0}}$. We now proceed to derive a contradiction from the assumption that $V^{G_{0}} \neq 0$.

This time, $V^{G_{0}}$ is a trivial $G_{0}$-module, so the action of $G_{0}$ on $V / V^{G_{0}}$ is through $\operatorname{det} \rho_{0}=1$, i.e., $V / V^{G_{0}}$ is also a trivial $G_{0}$-module. So $\rho_{0}$ is given by upper triangular matrices with 1 in the diagonal, i.e., its image is isomorphic to $\mathbf{Z}_{\ell}$. Let $F$ be the subfield of the separable closure of $K$ fixed by ker $\rho$ and $L$ the subfield of $F$ fixed by $G_{0}$. Then $L / K$ is an extension by constants and $F / L$ is a $\mathbf{Z}_{\ell^{-}}$extension, given by adjoining $\alpha_{n}^{1 / \ell^{n}},\left(\alpha_{n}\right)_{n} \in \lim L^{*} /\left(L^{*}\right)^{\ell^{n}}$. Since $L / K$ is Galois, the $\mathbf{Z}_{\ell^{-}}$line generated by $\left(\alpha_{n}\right)_{n}$ has to be $G_{K}$-invariant. It follows that $\rho$ can be put in upper-triangular form with the cyclotomic character $\chi$ in the diagonal (since it gives the action of Galois in $L / K$ ). Since, again, the determinant of $\rho$ is $\chi$, the other diagonal entry is 1 . Thus, the trace of $\rho$ applied to Frobenius at a place $v$ of $K$ is $1+N v$. On the other hand, since $\rho$ locally comes from an elliptic curve, this trace is an integer of absolute value at most $2(N v)^{1 / 2}$. Taking $N v$ sufficiently large gives a contradiction.

\section{REMARKS}

Let $K$ be a number field or a function field. One might consider extending the approach of [5] and the present paper using (subvarieties of) moduli spaces of abelian varieties of arbitrary dimension. This leads to the following. Consider a Galois representation $\rho: G_{K} \rightarrow G L_{n}\left(\mathbf{Z}_{\ell}\right)$, such that $\rho$ restricted to a decomposition group at $v$ corresponds to the action of $G_{K_{v}}$ on the Tate module $T_{\ell} E_{v}$ for abelian varieties $E_{v} / K_{v}$ of dimension $n$, with good reduction for $v \notin S$. In particular, this representation is unramified outside $S$. In the number field case, the Fontaine-Mazur conjecture [3] predicts that this representation occurs 
as a factor of the Galois representation of $G_{K}$ on $H^{i}\left(E, \mathbf{Q}_{\ell}\right)$ for some algebraic variety $A / K$ and some $i$. If that is indeed the case, then weight considerations and the fact the representation locally comes from an abelian variety imply that $i=1$. We may thus replace $A$ by its Albanese variety and assume, without loss of generality, that $A$ itself is an abelian variety. The representation $\rho$ corresponds to a Galois invariant factor $V$ of $T_{\ell} A$ such that for every place $v$ of $K$, there is an abelian variety $E_{v} / K_{v}$ such that the action of the absolute Galois group of $K_{v}$ on $V$ gives a representation isomorphic to $T_{\ell} E_{v}$. Does it follow that $A$ has a factor $E$ with $T_{\ell} E$ isomorphic to $V$ ? If that was the case, then we could extend much of our method. Unfortunately the answer to this question is no. The following counterexample is due to Y. Zarhin.

Let $A / K$ be a simple abelian surface (where $K$ is either a number field or a function field), whose ring of (absolute) endomorphisms is a maximal order in an indefinite quaternion algebra $D$ over the rationals. These are well-known to exist. For explicit examples, see e.g., [1]. Assume that $K$ is large enough so that all endomorphisms of $A$ are defined over $K, A$ has semistable reduction everywhere and, if $A$ has good reduction at $v$ then all the endomorphisms of its reduction $\tilde{A}_{v}$ are defined over the residue field. It is known that if $A(K)$ contains all points of order 12 on $A$ then $K$ is large enough in this sense. Note that $\mathbf{Q}$ is never large enough.

Then $A$ has good reduction at every $v$ and this reduction is isogenous to the square of an elliptic curve $\tilde{E}_{v}$. Now suppose that $D$ splits at

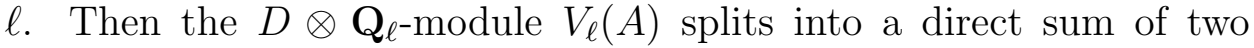
copies of a two-dimensional Galois invariant subspace $V$. If the residual characteristic of $v$ is not $\ell$, then one may identify the $\operatorname{Gal}\left(K_{v}\right)$-module $V$ with $V_{\ell}\left(E_{v}\right)$ where the elliptic curve $E_{v} / K_{v}$ is a lifting of $\tilde{E}_{v}$ to $K_{v}$.

This example also shows that without the non-integrality hypothesis in Theorem 1 the proof does not work. Indeed, the adelic point on a modular curve whose component at $v$ is $E_{v}$ corresponds to a global Galois representation and is thus unobstructed in the modular tower. But this representation does not come from a global elliptic curve, since this would force the abelian surface $A$ to be non-simple, contrary to the assumptions.

\section{ACKNOWLEDGEMENTS}

I would like to thank Y. Zarhin for the example in section 3, J.-M. Fontaine, M. Kisin and K. Ribet for discussions about the FontaineMazur conjecture, the University of Canterbury and IMPA for their 
hospitality and the NSA (grant MDA904-H98230-09-1-0070) and CAPES (bolsa PVE) for financial support.

\section{REFERENCES}

[1] J. D. Achter, Split reductions of simple abelian varieties, Math. Res. Lett. 16 (2009), 199-213.

[2] P. Deligne, Les constantes des equations fonctionnelles des fonctions L, in Modular Functions of One Variable II, Springer Lecture Notes in Mathematics 349 (1973) 501-597.

[3] J.-M. Fontaine, B. Mazur, Geometric Galois representations, in Elliptic curves, modular forms, \& Fermat's last theorem (Hong Kong, 1993), Int. Press, Cambridge, MA, (1995), pp 41-78.

[4] D. Harari, J. F. Voloch, The Brauer-Manin obstruction for integral points on curves, Math. Proc. Cam. Phil. Soc. 149 (2010), 413-421.

[5] D. Helm, J. F. Voloch, Finite descent obstruction on curves and modularity, BLMS, to appear.

[6] B. Poonen, J. F. Voloch: The Brauer-Manin obstruction for subvarieties of abelian varieties over function fields, Annals of Math., 171 (2010) 511-532.

[7] V. Scharaschkin: Local-global problems and the Brauer-Manin obstruction, 1999, Ph.D. thesis, University of Michigan.

[8] A. Skorobogatov, Torsors and rational points, Cambridge University Press, Cambridge 2001.

[9] M. Stoll, Finite descent and rational points on curves, Algebra and Number Theory 2 (2008), no 5, 595-611.

[10] C.-L. Sun, Adelic points of subvarieties of isotrivial semi-abelian varieties over a global field of positive characteristic. arXiv:1005.4998

Department of Mathematics, University of Texas, Austin, TX 78712, USA

E-mail address: voloch@math.utexas.edu

URL: http: //www.ma.utexas.edu/ voloch/ 